\title{
Alive but not well: the limited validity but continued utility of the concept of schizophrenia
}

\author{
Anthony W. Zoghbi and Jeffrey A. Lieberman* \\ Department of Psychiatry, Columbia University College of Physicians \& Surgeons, New York, NY
}

\begin{abstract}
Guloksuz \& van Os boldly challenge the status quo as pertains to schizophrenia. In 'The Slow Death of the Concept of Schizophrenia, and the Painful Birth of the Psychosis Spectrum' (Guloksuz \& van Os, 2017) they thoughtfully review long-standing concerns about this diagnostic category and present a new conceptualization. The authors question the validity of the schizophrenia concept citing variable clinical outcomes, transdiagnostic manifestations of psychosis, and the difficulty in identifying biomarkers, among other concerns. They also point toward the over-representation of schizophrenia in the psychosis literature and lament that patients and clinicians have come to associate this illness with predominantly poor outcomes. Finally, they propose removing the diagnosis of schizophrenia from the diagnostic nomenclature and instituting a broad new classification system, 'psychosis spectrum disorder' (PSD), to capture the many manifestations of psychosis. In this commentary, we advise against the institution of a psychosis spectrum due to the potential negative effects this framework would have on clinical care and progress in biological research.
\end{abstract}

Received 21 February 2017; Revised 30 March 2017; Accepted 19 June 2017; First published online 26 October 2017

Key words: Psychosis spectrum, schizophrenia, diagnosis, DSM.

In their article, "The slow death of the concept of schizophrenia and the painful birth of the psychosis spectrum," Guloksuz \& van Os carefully outline the shortcomings of the schizophrenia diagnosis and describe their rationale for moving towards a spectrum approach for psychosis (Guloksuz \& van Os, 2017). We agree that the diagnostic concept of schizophrenia is an imperfect one, and that there is a broad spectrum of disease severity and phenotypic manifestations. While creating a psychosis spectrum seems attractive and even justified on some grounds, there are significant limitations to such a model. Our greatest concerns are that it would misrepresent a transdiagnostic symptom as a unitary disease process, potentially hamper rather than aid research efforts, and disrupt patient care due to its lack of clinical utility. In this sense, the suggested PSD model while more circumscribed, suffers from similar shortcomings as the Research Domain Criteria (RDoC) proposed by Tom Insel when he led the NIMH (Insel \& Cuthbert, 2015). The impetus for RDoC was the lack of etiologic validity of DSM-5 diagnoses. However, diagnostic validity also derives from clinical validators, such as making correct prognoses, selecting correct treatments, and making correct predictions of outcome. Each of these medical activities serves as a key validator. A psychosis

* Address for correspondence: Jeffrey A. Lieberman, 1051 Riverside Drive, NY, NY 10032.

(Email: JLieberman@columbia.edu) spectrum would be as unlikely as RDoC is to validate the course and treatment of a given patient.

Guloksuz \& van Os cite the shift in the DSM-5 to spectrum-based diagnostics for autism and substance use disorders (SUDs) as evidence that diagnostic practice is moving generally towards a spectrum-based approach. However, there is a meaningful conceptual difference between moving these disorders onto a spectrum and doing the same for psychosis. In autism and SUDs, spectrums allow for a wider range of syndrome severity to be captured without changing the fundamental diagnostic criteria of the illnesses. However, depending on how the boundaries of the psychosis spectrum are drawn, the psychosis spectrum might change not only the boundaries of psychosis, but also primarily non-psychotic conditions, such as mood and personality disorders. It is well established that these conditions have distinct risk factors, co-morbidities, co-occurring cognitive and functional impairments, courses, and treatments. Would major depression or borderline personality disorder, which commonly feature psychotic symptoms (Ohayon \& Schatzberg, 2002; Schroeder et al. 2013), be re-classified as PSDs when patients manifest those symptoms? What would the implications be for clinical management if that were the case? And what would be the implications for the PSD system if certain psychiatric disorders with psychotic symptoms were left out of this spectrum?

Furthermore, creating a psychosis spectrum implies that psychosis itself is the disease. Unlike the syndrome 
of autism, psychosis is a transdiagnostic symptom that has psychiatric, neurologic, endocrine, infectious, and drug-induced etiologies. Though psychosis exists on a continuum of severity, which makes the spectrum approach appealing, this does not necessarily mean that it is caused by the same underlying disease process. Cough is an appropriate analogy. Cough is also a transdiagnostic symptom with diverse etiologies. We also know that different etiologies of cough result in different severities of the symptom: gastroesophageal reflux can cause a mild and transient cough and pertussis causes an intractable whooping cough. The treatment for the underlying cause of these two coughs is completely different. Though the authors acknowledge the likely 'existence of distinct diseases in the broad psychosis spectrum,' (Guloksuz \& van Os, 2017) creating a PSD construct would place too much emphasis on a single, non-specific symptom cluster while neglecting other important symptom domains. In the case of schizophrenia, the focus on psychosis would divert attention away from cognitive and negative symptoms domains, which are known to be the most disabling (Wieselgren et al. 1996; Green et al. 2004; Milev et al. 2005).

While we agree with the authors' that the limited validity of the schizophrenia diagnosis has likely slowed the pace of biologic research, we think that moving toward PSD system would exacerbate these issues. The authors state that 'an artificial categorization leads to a considerable loss of power and precision' and that 'a multimodal investigation of genetic susceptibility...not confounded by current diagnostic categories, would contribute to a better understanding of psychotic phenomena' (Guloksuz \& van Os, 2017). Yet, the proposition of a psychosis spectrum disorder might simply be a broader artificial categorization leading to further reductions in precision and statistical power.

Precise phenotypic definitions are important for research and particularly critical to genetic studies. While schizophrenia has a high heritability around 60-80\% (Kendler \& Diehl, 1993; Cannon et al. 1998), the heritability of milder or more transient psychotic disorders has not been systematically studied and might be far lower than schizophrenia. If these two syndromes were lumped together under the umbrella of PSDs, but in actuality represent distinct diseases, the resulting diagnostic misclassification would lead to a substantial reduction of statistical power (Wray et al. 2012). While one could reasonably argue that schizophrenia is a collection of distinct diseases, it is not clear how further broadening the psychosis phenotype would improve our ability to identify disease-associated mutations. We agree with the need to develop biologically based hypotheses not confined by the clinical boundaries of the DSM. However, for the reasons above, we do not feel that PSDs would enhance biologically based psychiatric research.

While we applaud their efforts for nosologic innovation, we worry that the risk of conflation of all psychotic disorders into one long spectrum is insufficiently justified. And while we recommend against adopting a spectrum approach for psychosis because of the potential negative implications for patient care, its narrow focus on a single symptom domain with diverse etiologies, and lack of an evidence base supporting its use in clinical or research settings, we encourage Guloksuz \& van Os and others to conduct the necessary research to answer these fundamental questions about the reliability, validity, and utility of the PSD classification system.

\section{References}

Cannon TD, Kaprio J, Lonnqvist J, Huttunen M, Koskenvuo M (1998). The genetic epidemiology of schizophrenia in a Finnish twin cohort. A population-based modeling study. Archives of General Psychiatry 55, 67-74.

Green MF, Kern RS, Heaton RK (2004). Longitudinal studies of cognition and functional outcome in schizophrenia: implications for MATRICS. Schizophrenia Research 72, 41-51.

Guloksuz S, van Os J (2017). The slow death of the concept of schizophrenia. Psychological Medicine 47, 1-16.

Insel TR, Cuthbert BN (2015). Medicine. Brain disorders? Precisely. Science 348, 499-500.

Kendler KS, Diehl SR (1993). The genetics of schizophrenia: a current, genetic-epidemiologic perspective. Schizophrenia Bulletin 19, 261-285.

Milev P, Ho BC, Arndt S, Andreasen NC (2005). Predictive values of neurocognition and negative symptoms on functional outcome in schizophrenia: a longitudinal first-episode study with 7-year follow-up. American Journal of Psychiatry 162, 495-506.

Ohayon MM, Schatzberg AF (2002). Prevalence of depressive episodes with psychotic features in the general population. American Journal of Psychiatry 159, 1855-1861.

Schroeder K, Fisher HL, Schafer I (2013). Psychotic symptoms in patients with borderline personality disorder: prevalence and clinical management. Current Opinion in Psychiatry 26, 113-119.

Wieselgren IM, Lindstrom E, Lindstrom LH (1996). Symptoms at index admission as predictor for 1-5 year outcome in schizophrenia. Acta Psychiatrica Scandinavica 94, 311-319.

Wray NR, Lee SH, Kendler KS (2012). Impact of diagnostic misclassification on estimation of genetic correlations using genome-wide genotypes. European Journal of Human Genetics 20, 668-674. 